\title{
Multiresolution analysis in statistical mechanics. II. The wavelet transform as a basis for Monte Carlo simulations on lattices
}

\author{
Ahmed E. Ismail, George Stephanopoulos, and Gregory C. Rutledge \\ Department of Chemical Engineering, Massachusetts Institute of Technology, \\ Cambridge, MA 02139
}

(October 29, 2018)

\begin{abstract}
In this paper, we extend our analysis of lattice systems using the wavelet transform to systems for which exact enumeration is impractical. For such systems, we illustrate a wavelet-accelerated Monte Carlo (WAMC) algorithm, which hierarchically coarse-grains a lattice model by computing the probability distribution for successively larger block spins. We demonstrate that although the method perturbs the system by changing its Hamiltonian and by allowing block spins to take on values not permitted for individual spins, the results obtained agree with the analytical results in the preceding paper, and "converge" to exact results obtained in the absence of coarse-graining. Additionally, we show that the decorrelation time for the WAMC is no worse than that of Metropolis Monte Carlo (MMC), and that scaling laws can be constructed from data performed in several short simulations to estimate the results that would be obtained from the original simulation. Although the algorithm is not asymptotically faster than traditional MMC, because of its hierarchical design, the new algorithm executes several orders of magnitude faster than a full simulation of the original problem. Consequently, the new method allows for rapid analysis of a phase diagram, allowing computational time to be focused on regions near phase transitions.
\end{abstract}

\section{INTRODUCTION}

One of the fundamental challenges in the simulation of very large systems is balancing the competing aims of accuracy, both numerical and physical, and computational efficiency. As the number of degrees of freedom, the number of interactions, and the number of system parameters become large, the computational cost and storage requirements of any algorithm which models these systems rapidly become prohibitive. For a system with $N$ degrees of freedom, the complexity of simulation algorithms is typically $O\left(N^{2}\right)$, although this can be reduced to $O(N)$ for methods incorporating both cell and Verlet lists ${ }^{1}$ and $O\left(N^{3 / 2}\right)$ for methods including Ewald sums, ${ }^{2}$ or increased to $O\left(N^{3}\right)$ or more for quantum methods. ${ }^{3}$ Consequently, very large systems are still expensive to simulate, even with efficient algorithms. One way to reduce the complexity of such systems is to employ a coarse-graining method, which systematically reduces the number of degrees of freedom in the system, and thereby the overall complexity of the simulation. Coarse-graining techniques have been developed for both on- and off-lattice calculations. Lattice simulations have generally relied on coarse-grainings based on renormalization group theory, ${ }^{4-6}$ while off-lattice simulations include coarse-graining techniques as varied as united-atom models, mapping to lattice models, ${ }^{7,8}$ dissipative particle dynamics, ${ }^{9}$ and bead-and-spring models. ${ }^{10,11}$

At the same time, any benefits obtained from applying a suitable coarse-graining technique must be weighed against the principal difficulty of using such a method-inaccurate numerical results, such as a different critical point. The use of uncontrolled approximations also presents a problem, since even if a simulation converges to give an answer, we often cannot use results from those simulations to provide any insight into the systems about which we are actually interested. In addition, in virtually all cases the transformation is irreversible: we cannot reconstruct our original system after we have "evolved" a coarse-grained system.

The work presented in this paper represents the application of a new technique to enhance the performance of traditional lattice simulations using the wavelet transform method. The theoretical foundations of this technique are outlined in the preceding paper (henceforth denoted as $\mathrm{I}^{12}$ ). First developed as an analysis technique which has found wide acceptance in signal processing, the wavelet transform has not yet been extensively applied to the field of molecular simulations. Exceptions to this include the variational work of Best and Schäfer, ${ }^{13,14}$ applied to statistical field theory, as well as the work of Huang in studying self-similarity in high-energy physics. ${ }^{15}$

We choose to study the class of Ising lattices with pairwise and external field interactions, so that the Hamiltonian can be written in the form

$$
\mathcal{H}=-\sum_{i} h_{i} \sigma_{i}-\sum_{i} \sum_{k} J_{i k} \sigma_{i} \sigma_{k}
$$

where the indices $i$ and $k$ run over all spins in the system, and the interaction strengths $h_{i}$ and $J_{i k}$ can vary with position in the lattice. The nearest-neighbor Ising lattice corresponds to $J_{i k}=0$ unless $\sigma_{i}$ and $\sigma_{k}$ are neighboring 
spins on the lattice, in which case $J_{i k}=J$. Our goal is to construct a phase diagram for such systems as efficiently as possible. The original problem would be studied using the Metropolis Monte Carlo (MMC) technique, along with possible improvements such as the Swendsen-Wang or Wolff spin-cluster algorithms. ${ }^{16,17}$ We introduce a new method for studying this problem, using the wavelet transform technique to simulate the system hierarchically, by systematically transforming (1) to provide expressions for potentials at coarser scales.

Since the systems under consideration in this paper are lattices, we present the wavelet transform using the discrete framework, involving filter banks and matrices. In the discussion below, we present equations for one-dimensional systems only; however, the simulations are performed using higher-dimensional wavelets created using the lifting scheme of Sweldens. ${ }^{18}$ The lifting scheme can be applied recursively as needed to achieve representations of the data at multiple length scales. However, we do not move between different physical models for the system, but only change the length scales which the different variables describe. As a result, the wavelet transform can be described as a multiresolution technique; ${ }^{18-21}$ multiresolution techniques comprise a subset of the more general class of multiscale techniques, which can describe any simulation involving multiple modeling steps, possibly involving the use of multiple underlying physical models. ${ }^{22,23}$ We refer the reader to paper I for further details on the implementation of the wavelet transform with respect to lattice systems.

\section{THE WAVELET-ACCELERATED MONTE CARLO (WAMC) ALGORITHM}

The principal difficulty of performing a wavelet transformation on a lattice system is in working with the discrete set of values that each spin is permitted to take, such as in a spin- $q$ Ising model. Because the transformed variables are linear combinations of the original variables, the constraint that the spins of the individual sites on the original lattice must be drawn from the set $\{-q,-q+1, \ldots, q-1, q\}$ quickly becomes a more complicated constraint on the transformed variables $\tilde{s}_{i}$ and $\tilde{\delta}_{i}$. As the system size becomes large, the difficulty of rewriting the spin constraints proves so great that previous investigations of the use of wavelets in statistical field theory ignored Ising models altogether. ${ }^{13}$ Consequently, we would like, if at all possible, to avoid computations involving original states after we have carried out the wavelet transformation. At this point, we take note of the application of wavelets to image compression, where the goal is to reduce the amount of information needed to reconstruct an image. We would like to apply this technique to lattice systems, and reduce the number of degrees of freedom which must be accounted for in our calculations.

We consider our system to be a $d$-dimensional regular lattice $\mathcal{L}$ with side length $l$, so that the size of the lattice is $N=|\mathcal{L}|=l^{d}$, and we let a site $\sigma_{i}$ on the lattice $\mathcal{L}$ be characterized by a "spin" chosen from a finite set $\mathcal{J}$ of values and by its physical location on the lattice. For the spin- $\frac{1}{2}$ Ising model, for example, the set $\mathcal{J}$ is just $\left\{+\frac{1}{2},-\frac{1}{2}\right\}$ (although for computational convenience this is usually treated as $\{+1,-1\}$, a convention which we follow below); similarly, for a lattice gas based on a spin-1 Ising model, $\mathcal{J}=\{0,1,2\}$ represents the allowed occupation numbers of each lattice site. We then assume that the only physical interactions that occur are either interactions with an external field $h_{i}$ which can vary at each lattice site, or pairwise interactions with the bilinear form $U\left(\sigma_{i}, \sigma_{j}\right)=J_{i j} \sigma_{i} \sigma_{j}$, where $J_{i j}$ is usually a function only of the spacing between sites $i$ and $j$. Consequently, the Hamiltonian of the system can be written in the form (1),

$$
-\beta \mathcal{H}=\sum_{i} h_{i} \sigma_{i}+\sum_{i} \sum_{j} J_{i j} \sigma_{i} \sigma_{j} .
$$

For the purposes of our simulations, however, we will find it more convenient to treat the set of spins $\left(\sigma_{1}, \ldots, \sigma_{N}\right)$ and the external field $\left(h_{1}, \ldots, h_{N}\right)$ as vectors $\mathbf{u}$ and $\mathbf{h}$, and the pairwise interaction strengths $J_{i j}$ as a matrix $\mathbf{J}$. Then the Hamiltonian (2) can be written in matrix form as

$$
-\beta \mathcal{H}=\mathbf{h}^{T} \mathbf{u}+\mathbf{u}^{T} \mathbf{J} \mathbf{u} .
$$

This formulation of the problem is similar in spirit to that of graph theory, where the pairwise potential $J_{i j}$ is used to generate an adjacency list which specifies which edges interact. ${ }^{24}$ Using (3) as the basis for a Monte Carlo simulation requires the calculation of the change of energy $\Delta E_{n m}$ from microstate $\mathbf{u}_{m}$ to microstate $\mathbf{u}_{n}$ :

$$
\Delta E_{n m}=\mathbf{h}^{T}\left(\mathbf{u}_{n}-\mathbf{u}_{m}\right)+\left(\mathbf{u}_{n}-\mathbf{u}_{m}\right)^{T} \mathbf{J}\left(\mathbf{u}_{n}-\mathbf{u}_{m}\right) .
$$

If moves are restricted to changes of single spin flips, then only a single entry of $\mathbf{u}_{n}-\mathbf{u}_{m}$ is nonzero, and therefore the calculation (4) reduces to a dot product, instead of a matrix multiplication.

As described in I, the action of the wavelet transform is to insert between each product in (3) or (4) the identity matrix in the form $\mathbf{I}=\mathbf{W}^{T} \mathbf{W}$, where $\mathbf{W}$ is the wavelet transform which maps data from one scale to the next 
coarser scale, containing half as many data points. The resulting expressions rewrite the Hamiltonian in terms of wavelet-transformed averages and differences, with downsampling needed to reduce the number of variables from $2 N$ to $N$. As before, the wavelet transform can be iterated by applying it to successive sets of averages, leading after $K$ iterations to Hamiltonians of the form:

$$
-\beta \tilde{H}=\left(\tilde{\mathbf{h}}^{(K)}\right)^{T} \tilde{\mathbf{u}}^{(K)}+\left(\tilde{\mathbf{u}}^{(K)}\right)^{T} \tilde{\mathbf{J}}^{(K)} \tilde{\mathbf{u}}^{(K)},
$$

where in (5) the $\tilde{\mathbf{u}}^{(K)}$ represent "block spins" whose values are determined by wavelet averaging over some well-defined region of the original system. The Hamiltonians (3) and (5) have the same formal structure, so that Monte Carlo simulations of the two systems are essentially identical. The only modifications needed to simulate a coarse-grained Hamiltonian are the ability to select new microstates $\tilde{\mathbf{u}}_{i}^{(K)}$ which are generated through wavelet transformations of the original microstates $\mathbf{u}_{i}$, and the elimination of unwanted degrees of freedom from (5). It should be noted that in $(5)$, the elements of $\tilde{\mathbf{u}}^{(K)}$ are not restricted to the same values as in the original system, but are free to take on any value which is consistent with the wavelet transform applied to the system.

From above, we saw that for even Hamiltonians $\tilde{H}(\tilde{\mathbf{x}})$, we should have that $\langle\delta\rangle_{\tilde{H}}=0$ for any wavelet difference $\delta$, where $\langle\cdot\rangle_{\tilde{H}}$ denotes the ensemble average weighted by the Hamiltonian $\tilde{H}$. As a "worst-case scenario" for our method, we shall assume not only that $\langle\delta\rangle_{\tilde{H}}=0$, but also that any terms in the Hamiltonian (5) containing fluctuation terms can be neglected as well. This assumption allows us to reduce the size of $\tilde{\mathbf{J}}{ }^{(K)}$ from $N \times N$ to $2^{-K d} N \times 2^{-K d} N$, where $d$ is the lattice dimensionality. Consequently, instead of performing calculations involving all of the original variables $\mathbf{x}$ which describe the state of our system, we consider functions only of local averages of our original variables. However, we anticipate that this simplification of the interactions present in the system will have a significant impact on the thermodynamic behavior of the resulting system; we illustrate these effects below.

To generate the new microstates $\tilde{\mathbf{u}}_{i}^{(K)}$, we need an estimate for the probability distribution $p\left(\tilde{u}_{i}^{(K)}\right)$ which describes the individual sites in the coarse-grained lattice. Determining the correct distribution for a given $\tilde{u}_{i}^{(K)}$ would require a detailed simulation of the original system. An alternative, ignoring the effect of neighboring block spins, would be to perform an exact enumeration of the spins within a block, which is possible only for the smallest of block spins. Since we would like to apply this method to systems of arbitrary size, we want to avoid both of these options. Therefore, we simulate a sublattice with the same dimensions as $\tilde{u}_{i}^{(K)}$, ignoring physical interactions with the rest of the system by using either free or periodic boundary conditions. Using the standard Metropolis acceptance criterion, we compute distributions for the properties of the small lattice, such as the magnetization. Then, according to the matrix formulation described in Sections II and III of Paper I, ${ }^{12}$ since the wavelet transform defines a single block spin $\tilde{u}_{i}^{(K)}$ as a linear function of the individual spins at level $K-1$ which it replaces, we can use the linearity properties of probability distributions to convert the distribution of the properties directly into a distribution for the block spin $\tilde{u}_{i}^{(K)}{ }^{25}$ Finally, using the distribution for the block spin $\tilde{u}_{i}^{(K)}$ as a starting point, we perform a Monte Carlo simulation on the system of block spins defined by the Hamiltonian (5).

Although (3) and (5) are structurally the same, we cannot impose a one-to-one correspondence between the states in the configuration space of (4) and the states in the configuration space of (5). Consequently, the thermodynamic information obtained from the two will not necessarily be identical; as we have shown in paper I, there is under fairly broad conditions a loss of entropy associated with the application of coarse-graining to a system. We can ensure that the detailed balance condition for the simulation based on (5) is satisfied for the new simulation by requiring

$$
\frac{\alpha\left(\tilde{\mathbf{u}}_{m}^{(K)} \rightarrow \tilde{\mathbf{u}}_{n}^{(K)}\right)}{\alpha\left(\tilde{\mathbf{u}}_{m}^{(K)} \rightarrow \tilde{\mathbf{u}}_{n}^{(K)}\right)}=\frac{p\left(\tilde{\mathbf{u}}_{n}^{(K)}\right)}{p\left(\tilde{\mathbf{u}}_{m}^{(K)}\right)} e^{-\beta\left(\mathcal{H}\left(\tilde{\mathbf{u}}_{n}^{(K)}\right)-\mathcal{H}\left(\tilde{\mathbf{u}}_{m}^{(K)}\right)\right)},
$$

where $\alpha(m \rightarrow n)$ is the probability of accepting a move from microstate $m$ to microstate $n$, and $p(m)$ is the probability of selecting microstate $m$ as determined from simulations on finer-grained lattices at lower scales.

\section{THEORETICAL PERFORMANCE OF WAMC VERSUS TRADITIONAL MC}

The wavelet transform is a hierarchical method which can be applied iteratively to a system to obtain successively coarser descriptions of a system. To describe the operation of the wavelet transform on a lattice model, we need to introduce some notation based on the various length scales in the problem. In the original problem, the applicable length scales are the lattice spacing $l$, the correlation length $\xi$, and the total lattice size $L$. Applying the wavelet 
transform method once increases the lattice spacing by some factor $a$, so that the ratios of correlation length to lattice spacing and of system length to lattice spacing each decrease by $a$. If we apply the wavelet transform $m$ times in succession, the corresponding factor becomes $a^{m}$.

We perform the simulation in a series of $K$ stages, where the length scales at each stage are functions of the length scales at the previous stages. The initial simulation is performed on a sublattice of the original problem, with lattice size $L^{(1)}<L$, where the superscript denotes the first stage of the simulation. The lattice spacing of the first stage is the same as in the original problem, so we define $l^{(1)}=l$. At each subsequent stage of the simulation, the lattice spacing of the $k$ th stage is defined by the recursive relation $l^{(k)}=L^{(k-1)} l^{(k-1)}$. Since $l^{(1)}$ is fixed to be the lattice spacing of the original lattice, the adjustable parameter in this relation is the lattice size $L^{(k)}$ of each stage. If we assume that the lattice is the same length in all dimensions at every stage, a single variable in stage $k$ is a block variable representing the $\left(L^{(k-1)}\right)^{d}$ variables simulated in stage $k-1$.

Assuming that the lattice is the same length in all directions both in the original problem and at every stage in the wavelet-transformed problems, there are $N_{t}=L^{d}$ lattice variables in the original problem, and $N^{(k)}=\left(L^{(k)}\right)^{d}$ lattice variables in the $k^{\text {th }}$ stage of the wavelet-transformed problem. However, each variable in stage $k$ is a block variable representing the average behavior of the $\left(L^{(k-1)}\right)^{d}$ variables in a block at stage $k-1$, so the number of total degrees of freedom represented at stage $k$ is $N_{t}^{(k)}=\prod_{i=1}^{k} N^{(i)}$, where $N_{t}^{(K)}=N_{t}$. The number of simulated degrees of freedom is $N_{s}=N_{t}$ for traditional Metropolis Monte Carlo (MMC), but $N_{s}=\sum_{i=1}^{K} N^{(i)}$ for WAMC. Because the running time of Monte Carlo simulations is usually linear in the number of degrees of freedom being simulated, the advantage of coarse-graining the system using a wavelet transform becomes evident. For example, consider an "original problem" of simulating a cubic lattice with 256 Ising variables on a side. If we divide the original problem into two stages consisting of cubes of 16 Ising variables on a side, we reduce the original problem of analyzing $256^{3}=$ $16,777,216$ variables to the simpler problem of analyzing $2\left(16^{3}\right)=8192$ variables. Although it is more difficult to produce a trial configuration in a simulation of the wavelet-transformed problem than in a simulation of the original Ising lattice problem, this is more than offset by the reduction in the number of degrees of freedom being simulated.

\section{RESULTS}

For the purposes of comparison, our "experimental" systems are two-dimensional Ising models of size $32 \times 32$, where we have run both MMC simulations on the full lattice, and WAMC simulations at a variety of resolutions; we shall denote these resolutions using the notation $(x, y)$, where $x$ indicates the length of the block size simulated in the first stage to estimate the probability distribution $p\left(\tilde{\mathbf{u}}^{(K)}\right)$ to be used in the second stage, and $y$ denotes the number of blocks on a side of the lattice in the second stage of the simulation.

\section{A. Order parameter}

Usually, the property of greatest interest in a simulation of a lattice system is the order parameter $\eta$. For spin systems, $\eta$ is generally taken to be the magnitude of the average magnetization, so that $\eta=\langle m\rangle$. [For $X Y$ and Heisenberg models, and other models where spins are oriented, we generally consider only the magnitude of the average vector $\eta=\langle m\rangle=\langle|\mathbf{m}|\rangle$.$] Generally, this is a very simple property to compute, since the value of the order$ parameter is constantly updated during the course of the simulation, and is thus always available.

For the $32 \times 32$ Ising model, the results of a MMC simulation, as well as $(4,8)$ - and $(8,4)$-WAMC simulations are shown as Figure 1. The primary difference in the curves for the three cases is that as the coarse-graining process decreases the number of degrees of freedom in the final stage of the simulation, the location of the Curie temperature, indicating onset of spontaneous magnetization, increases and the steepness of the curve below the Curie temperature decreases. This result is consistent with our findings for average absolute magnetization $\langle|m|\rangle$ from analytical models, discussed in paper I. In the present case, we note further that we achieve agreement between the different models not only in the low-temperature region, but also in the high-temperature regime $T \gg T_{c}$. The differences in the intermediate regime can be attributed largely to the difference in behavior that results from the use of the wavelet transform to move from the original Hamiltonian (3) to a coarse-grained Hamiltonian (5). Additionally, the increased noise in the WAMC results at intermediate and high temperatures arises because of the approximations used for the probability distributions $p\left(\mathbf{u}^{(K)}\right)$ at the second stage of the simulation. The relative lack of noise in the MMC results stem in part from the fact that the Metropolis technique leads to non-ergodic sampling of phase space as temperature increases, as the simulation tends to cycle through a limited number of states. ${ }^{26}$ 


\section{B. Internal Energy}

Plotting the internal energy $\langle U\rangle$ as a function of the temperature, we obtain curves that follow the same general pattern outlined in paper I. As illustrated in Figure 2, at low temperatures, the internal energy, as computed for the $32 \times 32$ model using standard MC as well as $(4,8)$ - and $(8,4)$-WAMC simulations, is in exact agreement for all methods. This occurs because only a few microstates of the system, corresponding to states that have all spins aligned, are actually observed by the system, and the wavelet transform preserves the energy of these states exactly. All three eventually reach an average internal energy of zero, but exact agreement is only expected in the infinite-temperature limit, when the difference in energy levels between microstates becomes unimportant. For intermediate temperatures, as before, the disagreement is a result of the change in form of the Hamiltonian that results from neglecting local correlations. Also, we note that for WAMC the "noise" in the internal energy increases both with increasing proximity to the "observed" critical point of the system as well as with increasing coarse graining. The additional coarse graining yields a Hamiltonian with reduced numbers of energy levels, since the energy of a block spin is defined here to be a function only of its overall magnetization, and not of its internal magnetization fluctuations; the reduced number of discrete energy levels yields noisier data.

\section{Fluctuation properties}

Fluctuation properties are useful for locating critical points since in the vicinity of a critical point, the magnitude of fluctuation properties is known to diverge as $t^{-\alpha}$, where $t \equiv\left|T-T_{c}\right| / T_{c}{ }^{27}$ Thus, a rapid increase in the value of a

fluctuation property such as the heat capacity at constant external field, $C_{H}=\left(\left\langle E^{2}\right\rangle-\langle E\rangle^{2}\right) / k_{B} T^{2}$, with respect to temperature can be used to estimate the critical temperature of a system. However, use of the wavelet transform leads to a decrease in the magnitude of the heat capacity, since the coarse-graining leads to smaller variances in the distribution of the energy $\langle U\rangle$. Consequently, the maximum value of the heat capacity $C_{\text {max }}$ decreases as a function of the number of degrees maintained in the problem.

In Figure 3, the heat capacity is shown as a function of dimensionless temperature $k_{B} T / J$ for the same systems as for the order parameter and internal energy measured above. We see that the location of the maximum of the heat capacity does increase, as expected. Although the relative maximum of the heat capacity obtained from the (4,8)and $(8,4)$-WAMC simulations appears to be identical, they differ by about 3 percent. Moreover, the actual value of the maximum is not as important as its existence and its location as a function of $k_{B} T / J$ and of the resolution of the model.

\section{Scaling results}

One application of the wavelet-accelerated MC method is to provide an upper bound for locating phase transitions. Running multiple simulations, at different levels of resolution, one can determine for each level of resolution the approximate phase transition temperature $T_{p}\left(N_{s}\right)$, where $N_{s}$ is the number of degrees of freedom (here, block spins) in the given system. From these data, one can extrapolate a scaling relationship of the form

$$
\left(T_{p}-T_{p}^{*}\right) \sim N_{s}^{-\gamma}
$$

where $\gamma$ is the corresponding "scaling" exponent, and $T_{p}^{*}$ is the phase transition temperature for the untransformed model. The estimate obtained for the scaling exponent $\gamma$ depends upon the technique used to calculate the transition temperature for a given system - for example, estimating divergence of the heat capacity versus the onset of spontaneous magnetization. Our simulations suggest that $\gamma$ is typically between 0.20 and 0.25 , with lower values obtained from divergence of heat capacity than from the onset of spontaneous magnetization.

As explained in I, using a relationship like (6) to estimate the phase transition temperature will usually lead to an overestimate of the phase transition temperature. This is a consequence of the underestimation of entropy that occurs through the reduction of the size of configuration space as a result of the wavelet transform. Estimates of $k_{B} T_{p} / J$, as determined by (6) for the two-dimensional Ising model considered here typically varied between about 2.7 and 2.9 , which is an error of approximately 25 percent from the theoretical value of 2.27 provided by the Onsager solution, but only about 20 percent from the results determined by the traditional MC simulations, which gave $k_{B} T_{p} / J \approx 2.35$. Although these errors are somewhat sizable, it is useful to note that the total computation time required to obtain the estimate using scaling laws is at least an order of magnitude smaller than the computation time required to perform a direct simulation on the original system. Thus, if computational time is at a premium, an effective approach may 
be to use the wavelet transform method to provide an upper bound for $T_{p}$, and then perform a direct simulation for the parameter space with temperatures below $T_{p}$.

\section{E. Decorrelation time}

Another important measure to study is the time required for decorrelated samples. It is well known that in the vicinity of the critical point, traditional Monte Carlo algorithms experience so-called "critical slowing-down." ${ }^{28}$ The Monte Carlo aspect of the WAMC algorithm does not vary from traditional Metropolis Monte Carlo, so we expect that the performance of the two algorithms should be similar, when measured near their respective critical temperatures.

To compare the two methods, we generated $2^{24}=16777216$ new configurations for the $32 \times 32$ Ising model at the critical point using traditional Metropolis Monte Carlo, as well as for the $(4,8)-,(8,4)-$, and $(16,2)$-WAMC models. To determine the correlation time, we used the blocking technique of Flyvbjerg and Petersen. ${ }^{29}$ The results are shown as Figures 4 and 5 for the MMC and (16,2)-WAMC models, respectively. The salient feature in the graph is the onset of a plateau in the value of the variance of the energy $\sigma_{E}^{2}$; according to the method of Flyvbjerg and Petersen, this indicates that configurations separated by a distance of $2^{x}$ steps are statistically independent, where $x$ is the number of blocking transformations that have been performed. For the MMC model, we find that $x \approx 13$ or $x \approx 14$ provides a decent estimate; for the (16,2)-WAMC model, $x=16$ is a good estimate for the index. [For the $(4,8)-$ and $(8,4)$-models (not shown), $x=15$ is a reliable estimate.] In each case, this indicates that between $2^{14}=16384$ and $2^{16}=65536$ steps are required between independent configurations. Thus, we conclude that there is no degradation of performance near the critical point of a WAMC simulation, relative to traditional MMC simulations.

\section{ANALYSIS}

\section{A. Measured performance comparison}

In comparing the performance of the standard Monte Carlo algorithm to the wavelet-accelerated Monte Carlo algorithm, we performed $5 \times 10^{5}$ lattice passes on a $32 \times 32$-lattice on a $733 \mathrm{MHz}$ Pentium II: for the standard Monte Carlo algorithm, this meant that, on average, $5 \times 10^{5}$ attempts were made to flip each spin. For the WAMC algorithm, $5 \times 10^{5}$ attempts were made to flip a spin on a given level. The results are summarized in Table 1 . We see that the $(4,8)$ - and $(8,4)$-simulations, which have the smallest total number of lattice sites $(80)$, perform the fastest; however, even the (16,2)-simulation, which has one-fourth as many variables (260) as the $32 \times 32$ standard Monte Carlo simulation (1024), finishes in less than 8 per cent of the time required for the latter simulation. As the system size increases, the computational efficiency achieved by breaking down the system into multiple stages, all of relatively equal size, becomes even greater: for a $128 \times 128$-lattice, the performance gain increases from a factor of approximately 25 to a factor of approximately 50, when we compare the $(32,4)$ - and $(16,8)$-WAMC simulations to the standard MC model. However, for the $(8,16)$-WAMC model, the complexity of assigning one of 65 possible values to each of 256 variables according to the correct probability distribution becomes comparable to that of the original problem, so that in fact standard MC runs in roughly a factor of 3 faster than the $(8,16)$-model.

Similar results are also observed for calculating the phase diagram of a $64 \times 64$-Ising lattice, which is shown in Figure 6 as a plot of average magnetization as a function of temperature and external field strength using an $(8,8)$ model, for temperatures between $T=0.5$ and $T=5.0$, and for field strengths between $h=-1$ and $h=1$. The phase diagram reproduces the essential features of the original two-dimensional ferromagnetic Ising lattice, such as the phase separation at $h=0$, although the exact shape differs from the results obtained via a standard Metropolis Monte Carlo simulations. However, the plot based on WAMC calculations is created approximately 40 times faster than would a comparable plot using standard MMC.

\section{B. Comparison with renormalization group methods}

Our observations also indicate that the accuracy of the wavelet-accelerated Monte Carlo simulations depends on the relative proximity to an "attractive fixed point" of the physical model in parameter space. Borrowed from renormalization group theory, these attractive fixed points represent the limiting behavior of the system under various conditions, such as the zero- and infinite-temperature limits and the limits of zero and infinite external field. As we approach these limiting cases, the approximations made in obtaining our wavelet-transformed Hamiltonian become increasingly less significant. 
Combining these observations allows us to design an on-line fine-tuning algorithm for the coarse graining of our system: the further away from the critical point of the parameter space, the smaller the number of degrees of freedom $N^{(K)}$ necessary to simulate the system must be. Thus, if we keep track of changes in fluctuation properties such as the heat capacity or the magnetic susceptibility as we change the system parameters $(h, T)$, we can get an estimate of our relative distance to the critical point. If we are sufficiently far away from the critical point, we can choose either to increase the number of stages $K$ that we simulate, or we can look at more degrees of freedom at lower stages by increasing $N^{(1)}, N^{(2)}, \ldots, N^{(K-1)}$. As we approach the critical point, we can either decrease the number of stages $K$ or include more degrees of freedom at higher stages by reducing $N^{(1)}, \ldots, N^{(K-1)}$.

\section{Sources of error}

In general, the source of our errors can be traced to the assumption that local fluctuation terms could be reasonably ignored in our coarse-grained Hamiltonian (5). This naive but otherwise useful assumption yields correct thermodynamic behavior when the overall physics of the system is particularly simple: in the low- and high-temperature regimes, for instance, when the number of observed microstates is small or when the differences between observed microstates is inconsequential. For more complicated behaviors, as found at intermediate temperatures and above all in the vicinity of a critical point, the use of this assumption has a drastic effect on both the phase space of the system, which in turn affects all thermodynamic properties of the system, including the internal energy and entropy of the system, as well as fluctuation properties of the system.

More complicated methods for dealing with fluctuation terms have significant drawbacks associated with them. Treating the fluctuation terms just like the block averages maintained in $\tilde{\mathbf{u}}^{(K)}$ means that the wavelet-transformed Hamiltonian is no simpler than the original Hamiltonian, which affords few advantages in computational time. Likewise, other approaches, such as parametrizing the probability distribution for the elements of $\tilde{\mathbf{u}}^{(K)}$ using a property like the energy $E$, introduce new functional dependencies which cannot be taken into account using the wavelet transform. Thus, we sacrifice one of the major advantages of the method-moving from one level to another is achieved exclusively through use of the wavelet transform. Thus, the most promising avenue for dealing with fluctuation terms is to develop a probability distribution for the fluctuation terms via the same approach used to determine probability distributions for the local averages. Then, using the probability distribution for the fluctuation terms, we can treat the discarded terms of the Hamiltonian as a noise term which can be used to restore some of the entropy that was lost as a result of the coarse-graining [see paper I for more details]. However, we have presented our results here to show what can be achieved under "worst-case" conditions, without the use of inverse coarse-graining methods.

Many coarse-graining techniques control errors by fitting the parameters of a new Hamiltonian to ensure agreement with some known structural information about the system, such as the radial distribution function. ${ }^{30,31}$ For lattice systems, this iterative approach is reflected in renormalization group theory, and notably the Wilson recursion method, ${ }^{32}$ which finds the fixed points of the system. As formulated, WAMC creates a coarse-grained Hamiltonian by truncating the Hamiltonian obtained after application of the wavelet transform. As an improvement to this, it should be possible to use the wavelet transform to determine which terms will appear in the Hamiltonian, and then determine the appropriate parameters to ensure the best fit for some desired property of the system using an iterative approach. ${ }^{33-35}$

\section{Constructing an adaptive algorithm for MC using the wavelet transform}

As pointed out above, the wavelet transform method tends to produce overestimates for the critical point of the system; therefore, if we start with the high-temperature limit of our algorithm and slowly reduce the temperature in our simulation, we can observe the movement toward the critical point by watching various fluctuation parameters,

such as the heat capacity $C_{H}=\left(\left\langle E^{2}\right\rangle-\langle E\rangle^{2}\right) / k_{B} T^{2}$. Near the critical point, we expect to see a rapid increase in the value of $C_{H}$, consistent with the expected logarithmic divergence observed in the limit of finite-size systems. ${ }^{3,27}$ If we use the onset of this logarithmic divergence as an indicator, we can then "step down" and use a finer lattice including more degrees of freedom. This system will naturally better reflect the physics of our system, particularly in the vicinity of the critical point. We expect that very near the critical point, we will have to simulate the system at the original scale, since this will be effectively the only level which accurately represents the underlying behavior of the system. However, the region of parameter space where this is necessary is relatively small compared to the complete parameter space. This is especially true when we consider that as we proceed below the critical temperature of the system, the logarithmic divergence of $C_{H}$ will also vanish. As a result, as we move increasingly far away from the critical point, we begin to approach the other fixed-point behaviors associated with the low-temperature limits 
of the system. Since these are reasonably well-preserved using the wavelet transformation, we can safely return to increasingly coarse-grained descriptions of our system as the simulation proceeds past the critical point.

As an example, we compute the spontaneous magnetization curve for a $64 \times 64$ Ising lattice in the temperature range $0.5 \leq T \leq 10.0$, with $\Delta T=-0.05$, and choosing as our refinement criterion $\Delta C_{H} / \Delta T \leq-0.5$, until we reach the finest scale, corresponding to the original problem. We begin by coarse-graining the system to an $(8,8)$ model, where we find that the criterion is triggered only at $T=5.1$; we then continue with a $(4,16)$-model, down to $T=4.0$, at which point the refinement criterion is exceeded. Refining once more, we proceed with a $(2,32)$-model until $T=3.4$, at which point the threshold is again crossed. Since the next refinement is the original problem, we proceed at this level of resolution until we have passed the critical point, so that $\Delta C_{H} / \Delta T$ is positive. As a coarsening criterion, we select for simplicity the opposite of the refinement criterion, $\Delta C_{H} / \Delta T \leq 0.5$. Using this criterion, we find that we coarsen the model to the $(2,32)-,(4,16)$ - and $(8,8)$-models at temperatures of $T=1.75, T=1.65$, and $T=1.55$, respectively. The rapid coarsening of the model results from the higher estimates of the critical point in the coarsened models. Since we are well past the critical point, we expect changes in the heat capacity as a function of temperature to be relatively small, and thus it is possible to obtain accurate results from a relatively coarse model. Computationally, the time required to create this diagram was only 28 per cent that required to perform a standard Metropolis Monte Carlo simulation with the same number of steps. Moreover, in the regions that were not simulated using MMC, the computation time required was just 8 per cent of the time required for MMC. The resulting plot of magnetization versus temperature, shown as Figure 7, compares favorably to the analytical solution of Onsager, which is also shown. ${ }^{36}$

\section{CONCLUSIONS}

The WAMC algorithm can dramatically reduce the time required to calculate the thermodynamic behavior of a lattice system; the trade-off for these savings in time is in the accuracy of the results obtained, a general feature of coarse-graining techniques. The error that results is a function of position in parameter space: the results obtained are generally accurate in the vicinity of fixed attractors of the system, and decrease as one approaches critical points of the parameter space. Near critical points, deviations from results performed on the original lattice system are the result of coarse-graining the Hamiltonian by eliminating local fluctuation terms. Consequently, this suggests that a hierarchical simulation which uses fluctuation properties such as the heat capacity $C_{H}$ to gauge proximity to critical points in "real time" would yield dramatic savings in the computation time of the behavior of a lattice system over a wide region of phase space, as regions of space close to fixed attractors would be simulated at a very coarse scale, with full-detailed simulations reserved only for regions of parameter space close to critical points.

\section{ACKNOWLEDGMENTS}

Funding for this research was provided in part by a Computational Sciences Graduate Fellowship (AEI) sponsored by the Krell Institute and the Department of Energy.

[1] L. Verlet, Phys. Rev. 159, 98 (1967).

[2] P. P. Ewald, Ann. Phys. 64, 253 (1921).

[3] D. Frenkel and B. Smit, Understanding Molecular Simulation: From Algorithms to Applications, Academic Press, San Diego, 1996.

[4] S.-K. MA, Phys. Rev. Lett. 37, 461 (1976).

[5] R. H. Swendsen, Phys. Rev. Lett. 42, 859 (1979).

[6] S. Curtarolo and G. Ceder, Phys. Rev. Lett. 88, 255504 (2002).

[7] J. Cho and W. L. Mattice, Macromolecules 30, 637 (1997).

[8] P. Doruker and W. L. Mattice, Macromolecules 30, 5520 (1997).

[9] A. G. Schlijper, P. J. Hoogerbrugge, and C. W. Manke, J. Rheol. 39, 567 (1995).

[10] W. Tschöp, K. Kremer, J. Batoulis, T. Bürger, and O. Hahn, Acta Polymer. 49, 61 (1998).

[11] W. Tschöp, K. Kremer, O. Hahn, J. Batoulis, and T. Bürger, Acta Polymer. 49, 75 (1998).

[12] A. E. Ismail, G. C. Rutledge, and G. Stephanopoulos, Submitted to J. Chem. Phys. (2002). 
[13] C. Best, A. Schäfer, and W. Greiner, Nucl. Phys. B: Proc. Suppl. 34, 780 (1994).

[14] C. Best and A. SchäfER, Variational Description of Statistical Field Theories Using Daubechies' Wavelets, http://xxx.lanl.gov/abs/hep-lat/9402012, 1994.

[15] D.-W. Huang, Phys. Rev. D 56, 3961 (1997).

[16] R. H. Swendsen and J.-S. Wang, Phys. Rev. Lett. 58, 86 (1987).

[17] U. Wolff, Phys. Rev. Lett. 62, 361 (1989).

[18] W. Sweldens, SIAM J. Math. Anal. 29, 511 (1997).

[19] S. G. Mallat, IEEE Trans. Pattern Analysis and Machine Intelligence 11, 674 (1989).

[20] S. G. Mallat, Trans. Amer. Math. Soc. 315, 69 (1989).

[21] I. Daubechies and W. Sweldens, J. Fourier Anal. Appl. 4, 247 (1998).

[22] J. Q. Broughton, F. F. Abraham, N. Bernstein, and E. Kaxiras, Phys. Rev. B 60, 2391 (1999).

[23] R. M. Nieminen, J. Phys.: Cond. Matt. 14, 2859 (2002).

[24] T. H. Cormen, C. E. Leiserson, and R. L. Rivest, Introduction to Algorithms, McGraw Hill-Mit Press, Cambridge, MA, 1990.

[25] P. Billingsley, Probability and Measure, Wiley Interscience, New York, 1995.

[26] D. P. Landau and K. Binder, A Guide to Monte Carlo Simulations in Statistical Physics, Cambridge University Press, Cambridge, 2000.

[27] R. K. Pathria, Statistical Mechanics, Butterworth-Heinemann, Woburn, MA, 1996.

[28] J. J. Binney, N. J. Dowrick, A. J. Fisher, and M. E. J. Newman, The Theory of Critical Phenomena: An Introduction to the Renormalization Group, Oxford University Press, Oxford, 1993.

[29] H. Flyvbjerg and H. G. Petersen, J. Chem. Phys. 91, 461 (1989).

[30] J. Baschnagel, K. Binder, P. Doruker, A. A. Gusev, O. Hahn, K. Kremer, W. L. Mattice, F. Müller-Plathe, M. Murat, W. Paul, S. Santos, U. W. Suter, and V. Tries, Adv. Poly. Sci. 152, 41 (2000).

[31] R. L. C. Akkermans and W. J. Briels, J. Chem. Phys. 114, 1020 (2001).

[32] K. J. Wilson and J. Kogut, Phys. Rep. 12, 75 (1974).

[33] A. P. Lyubartsev and A. Lahksonen, Phys. Rev. E 52, 3730 (1995).

[34] A. P. Lyubartsev and A. Lahksonen, Phys. Rev. E 55, 5689 (1997).

[35] G. C. Rutledge, Phys. Rev. E 63, 021111 (2001).

[36] L. Onsager, Phys. Rev. 65, 117 (1944).

FIG. 1. Absolute average magnetization as a function of the dimensionless temperature $k_{B} T / J$ for the $32 \times 32$ Ising model computed using standard MC (left curve), a (4,8)-WAMC simulation (center), and a $(8,4)$-WAMC simulation (right).

FIG. 2. Internal energy as a function of the dimensionless temperature $k_{B} T / J$ for the $32 \times 32$ Ising model computed using standard MC (left curve), a (4,8)-WAMC simulation (center), and a (8,4)-WAMC simulation (right).

FIG. 3. Heat capacity as a function of the dimensionless temperature $k_{B} T / J$ for the $32 \times 32$ Ising model computed using standard MC (left curve), a (4,8)-WAMC simulation (center), and a (8,4)-WAMC simulation (right).

FIG. 4. Graph showing variance in the estimate of energy as calculated using the method of Flyvbjerg and Petersen ${ }^{29}$ for a $32 \times 32$ Ising model measured at its critical temperature.

FIG. 5. Graph showing variance in the estimate of energy as calculated using the method of Flyvbjerg and Petersen ${ }^{29}$ for a $32 \times 32$ Ising model in a $(16,2)$-WAMC measured at the critical temperature determined from the simulation.

FIG. 6. Phase diagram plotting average magnetization versus temperature and external field strength for a $64 \times 64$ ferromagnetic Ising lattice, computed using an $(8,8)$-model via WAMC. The general features correspond to those that would be produced with standard MMC, but require less than 3 per cent of the computational time. 
FIG. 7. Phase diagram plotting average magnetization versus temperature for a $64 \times 64$ ferromagnetic Ising lattice, created using an adaptive WAMC algorithm, with refinement and coarsening criterion established using the change in heat capacity with respect to temperature $\Delta C_{H} / \Delta T$. The squares represent the simulation results, while the line reproduces Onsager's analytical result for the two-dimensional Ising model with zero external field. The simulation used in a given temperature region is shown on the plot. 
Table 1. Performance comparison for Metropolis Monte Carlo (MMC) versus Wavelet-Accelerated Monte Carlo (WAMC) algorithms

\begin{tabular}{|l|l|}
\hline Simulation & Time for $5 \times 10^{5}$ passes $(\mathrm{s})$ \\
\hline
\end{tabular}

\begin{tabular}{|l|l|}
\hline $32 \times 32$ MMC & 1824.22 \\
\hline$(4,8)-W A M C$ & 64.5781
\end{tabular}

\begin{tabular}{|l|l|l}
\hline$(4,8)$-WAMC & 64.5781 \\
\hline
\end{tabular}

\begin{tabular}{|l|l|l}
\hline$(8,4)$-WAMC & 55.1094 \\
\hline
\end{tabular}

\begin{tabular}{|l|l|l|l}
\hline$(16,2)-W A M C$ & 144.516 \\
\hline
\end{tabular} 
This figure "fig1.png" is available in "png" format from: http://arxiv.org/ps/physics/0212067v1 
This figure "fig2.png" is available in "png" format from: http://arxiv.org/ps/physics/0212067v1 
This figure "fig3.png" is available in "png" format from: http://arxiv.org/ps/physics/0212067v1 
This figure "fig4.png" is available in "png" format from: http://arxiv.org/ps/physics/0212067v1 
This figure "fig5.png" is available in "png" format from: http://arxiv.org/ps/physics/0212067v1 
This figure "fig6.png" is available in "png" format from: http://arxiv.org/ps/physics/0212067v1 
This figure "fig7.png" is available in "png" format from: http://arxiv.org/ps/physics/0212067v1 